\title{
Aberrant DNA methylation of T-cell leukemia, homeobox 3 modulates cisplatin sensitivity in bladder cancer
}

\author{
YASUHIRO TADA $^{1}$, AKIRA YOKOMIZO ${ }^{1}$, MASAKI SHIOTA ${ }^{1}$, \\ TOSHIYUKI TSUNODA ${ }^{1}$, CHRISTOPH PLASS ${ }^{2,3}$ and SEIJI NAITO ${ }^{1}$
}

\author{
${ }^{1}$ Department of Urology, Graduate School of Medical Sciences, Kyushu University, Fukuoka, Japan; \\ ${ }^{2}$ Department of Molecular Virology, Immunology, and Medical Genetics, Division of Human Cancer \\ Genetics, The Ohio State University, Columbus, OH 43220, USA; ${ }^{3}$ German Cancer Research \\ Center, Division of Epigenomics and Cancer Risk Factors, Heidelberg, Germany
}

Received February 4, 2011; Accepted April 12, 2011

DOI: $10.3892 /$ ijo.2011.1049

\begin{abstract}
The development of resistance to cisplatin during treatment of bladder cancer constitutes a major obstacle to curing bladder cancer. The identification of epigenetic biomarker molecules for cisplatin resistance and the elucidation of the function of the identified genes in bladder cancer will provide useful information. We found that the candidate gene $T L X 3$ was unmethylated in cisplatin sensitive cells and methylated in resistant cells. The suppression of TLX3 expression using TLX3-specific shRNA in parental cells increased cisplatin resistance. Contrarily, overexpression of TLX3 in resistant cells induced increased sensitivity to cisplatin. We found that $22(21 \%)$ out of 110 clinical samples of bladder cancer showed the methylated pattern using the COBRA assay in $T L X 3$. We found a correlation between $T L X 3$ methylation and the sensitivity to cisplatin in the clinical samples by SDI test. Cisplatin sensitivity was closely associated with the methylation status of $T L X 3$. These findings showed that the $T L X 3$ methylation may be useful as a novel biomarker for cisplatin resistance and can be used to design therapies to counteract the resistance against cisplatin in bladder cancer.
\end{abstract}

\section{Introduction}

$T L X 3$ belongs to a family of orphan homeobox genes that encode DNA-binding nuclear transcription factors. Homeobox genes are highly conserved master regulators that play a role in morphogenesis and cell differentiation. A number of homeobox genes including $T L X 3$ have been implicated in oncogenesis and prognosis in T-ALL. However, the roles of homeobox genes in solid tumor are still largely unknown $(1,2)$. Wang

Correspondence to: Dr Seiji Naito, Department of Urology, Graduate School of Medical Sciences, Kyushu University, 3-1-1 Maidashi, Higashi-ku, Fukuoka 812-8582, Japan

E-mail: naito@uro.med.kyushu-u.ac.jp

Key words: bladder cancer, cisplatin, DNA methylation, TLX3 et al reported that TLX3 expression is regulated through epigenetic mechanisms in prostate cancer cells (3).

Methylation of $\mathrm{CpG}$ islands is frequently associated with gene suppression. Epigenetic changes, such as aberrant DNA methylation, are the most frequent alterations found in human malignant tumors including bladder cancer (4-6). Epigenetic alterations play a major role in driving tumor initiation and progression. The main processes that contribute to the epigenome of cells are DNA methylation and histone modifications. However, $\mathrm{CpG}$ islands (CGI), most of which are located within or near regulatory regions of genes, seem to be protected from this modification in normal somatic cells (7). Accumulating evidence indicates that CGI methylation within promoters of specific tumor suppressor genes is associated with transcriptional silencing that compromises control of cell proliferation (8). Thus, aberrant methylation is now being investigated as a potential biomarker and for the development of pathway-specific therapeutic targets $(9,10)$. Furthermore, DNA methylation plays the key role of in bladder cancer pathogenesis and treatment decisions $(3,11,12)$.

Cisplatin, a platinum coordinated complex, is a widely used antineoplastic agent for the treatment of advanced bladder cancer as combination chemotherapy (M-VAC and GC therapies) and many other solid tumors. The development of resistance against cisplatin during treatment is common and constitutes a major obstacle to successful treatment outcome. Better biomarkers and an improved understanding of the underlying mechanisms of cisplatin resistance associated with bladder cancer will provide useful information for clinical decision making. However, biomarkers that can predict cisplatin resistance in bladder cancer patients have not been determined.

Here, we performed restriction landmark genome scanning (RLGS) to identify novel biomarkers of cisplatin resistance in bladder cancer based on DNA methylation analysis (13-15). TLX3 knockdown in cisplatin-sensitive cells by TLX3-specific shRNA showed increased resistance against cisplatin. TLX3 overexpression in cisplatin-resistant cells showed increased sensitivity to cisplatin. We found a relationship between cisplatin resistance as assayed by succinate dehydrogenase inhibition (SDI) test and the TLX3 methylation status in 
clinical samples of bladder cancer. We suggest that aberrant $T L X 3$ methylation is a novel biomarker to predict the sensitivity of cisplatin in bladder cancer and one of the mechanisms to develop cisplatin resistance.

\section{Materials and methods}

Cell line. Human bladder cancer T24 and KK47 cells were cultured in the MEM media (Invitrogen, Carlsbad, CA, USA), containing $10 \%$ fetal bovine serum. Human bladder cancer cisplatin-resistant cell lines were established by continuous exposure of T24 and KK47 cells to increasing concentrations of cisplatin and cultivated as previously described $(16,17)$. The resistance to cisplatin of T24DDP10 and KK47DDP20 cells was 8.4- and 18.7-fold compared with that of the parental cells, respectively $(16,17)$.

Patients and samples. In this study, 110 clinical samples with bladder cancer that underwent resection of bladder cancer in Kyushu University Hospital between September 1991 and June 2007 were studied. Tumor tissue samples were obtained under an Institutional Review Board-approved protocol, with subjects providing informed consent.

Isolation of DNA and RNA. DNA was isolated from the tissues of patients, using the Easy DNA Kit (Invitrogen) according to the manufacturer's protocol. RNA was isolated using the RNA extraction reagent, TRIzol (Invitrogen) according to the manufacturer's protocol.

Restriction landmark genomic scanning (RLGS). RLGS was performed as previously described (18). RLGS profiles of bladder cancer cells were visually inspected for differences in the presence and/or intensity of radiolabeled fragments. The use of cisplatin-resistant cells derived from the parental cells ensured that DNA polymorphisms that might be present at any of the restriction enzyme recognition sites would not introduce a bias in the analysis. RLGS fragments were cloned using the NotI/EcoRV boundary library mixing gels (19). All sequencing was performed at the Genotyping and Sequencing Unit at The Ohio State University, Division of Human Cancer Genetics. Southern hybridization was performed as previously described to confirm the methylation (19).

Bisulfite genomic sequencing and combined bisulfite restriction analysis (COBRA). Genomic DNA $(1 \mu \mathrm{g})$ was treated with sodium bisulfite according to published protocols with modifications (20). Primers were designed to allow amplification of both methylated as well as non-methylated DNA. The TLX3 forward primer was 5'-TTTTTGGTAAAGTTTTAGTG-3' and the reverse primer was 5'-ACCCTCCCAAATAACTAAC-3'. The reaction conditions had an initial denaturation step for $10 \mathrm{~min}$ at $95^{\circ} \mathrm{C}, 40 \mathrm{cycles}$ of $95^{\circ} \mathrm{C} \times 30 \mathrm{sec}, 52^{\circ} \mathrm{C} \mathrm{x} 30 \mathrm{sec}$, $72^{\circ} \mathrm{C}$ x $30 \mathrm{sec}$, and a final extension step at $72^{\circ} \mathrm{C}$ x $10 \mathrm{~min}$. For bisulfite sequencing, the PCR product was purified from a 2\% agarose gel using the Qiagen Gel Extraction kit (Qiagen, Valencia, CA, USA) according to manufacturer's protocol and subcloned using the TOPO TA-Cloning kit (Invitrogen). Four clones for each sample were sequenced. For combined bisulfite restriction analysis (COBRA), the bisulfite-treated DNA was
PCR-amplified. The PCR product was digested by Bst UI restriction enzyme and separated on an $8 \%$ polyacrylamide gel.

Semiquantitative RT-PCR analysis. The TLX3 forward primer was 5'-GCGCATCGGCCACCCCTACCAGA-3' and the reverse primer was 5'-CCGCTCCGCCTCCCGCTCCTC-3' (21). The reaction conditions had an initial denaturation step for $10 \mathrm{~min}$ at $95^{\circ} \mathrm{C}, 35$ cycles of $95^{\circ} \mathrm{C}$ x $30 \mathrm{sec}, 55^{\circ} \mathrm{C}$ x $30 \mathrm{sec}, 72^{\circ} \mathrm{C}$ x $30 \mathrm{sec}$, and a final extension step at $72^{\circ} \mathrm{C} \times 10 \mathrm{~min}$. The PCR products were separated by electrophoresis on $2 \%$ agarose gels, which were then stained with ethidium bromide. GAPDH was used as positive control for RT-PCR (data not shown). The GAPDH forward primer was 5'-ACCCAGAAGACTGTGGATGG-3' and the reverse primer was 5'-AGGGGTCTACATGGCAACTG-3'.

Plasmid construction. The CMV6-TLX3 plasmid expressing TLX3 protein and the CMV6 (control) plasmid were purchased from OriGene (Rockville, MD, USA). TLX3-specific shRNA plasmid (TLX3-shRNA) and negative control shRNA (controlshRNA), were purchased from OriGene.

Antibodies, transfections and immunobloting. Antibody against TLX3 (MO2) was purchased from (Abnova, Taipei, Taiwan). Anti- $\beta$-actin antibody was purchased from Sigma (St. Louis, MO). T24 cells were transiently transfected using Lipofectamine 2000 (Invitrogen) according to the manufacturer's protocol. T24DDP10 cells were transiently transfected using PolyFect transfection reagent (Qiagen) according to the manufacturer's protocol. Immunoblotting was performed using previously reported method $(22,23)$.

Drug sensitivity test. Drug sensitivity tests were done using previously reported method $(22,23)$. All assays were performed in quadruplicate, and the mean values $( \pm \mathrm{SD})$ were determined from three separate experiments. The results are shown as the percentage of control, while the absorbance of the untreated cells was considered to be $100 \%$.

Cell proliferation assay. Cell proliferation assay was done using previously reported method $(22,23)$. The results are representative of at least three independent experiments.

Succinate dehydrogenase inhibition (SDI) test. The SDI test was carried out using reported methods $(5,24)$. The succinate dehydrogenase (SD) activity, determined by 2,3,5-triphenylterazolium chloride as terazolium salt, was used as the predictor of cell viability. The chemosensitivity to cisplatin is given as a percentage of the SD activity in cisplatin-treated cells compared to non-treated cells.

Statistical analysis. For statistical analysis of drug sensitivity test and cell proliferation assay, we used Student's t-test. Mann-Whitney tests were used to compare two groups of $T L X 3$ methylation status with SD activity. Computations were carried out using StatView software (Tokyo, Japan).

\section{Results}

Altered gene methylation between cisplatin-sensitive and -resistant bladder cancer cells. RLGS was performed on 
Table I. Sequence characteristics of cloned RLGS spots lost in cisplatin resistant cells.

\begin{tabular}{llccc}
\hline RLGS fragment & \multicolumn{1}{c}{ Chromosomal location } & Cytogenetic mapping & Gene homology & CpG island location \\
\hline 3B56 & chr2:162243949 & $2 q 24.2$ & SLC4A10 & $5^{\prime}$ \\
4B33 & chr18:60874854 & $18 \mathrm{q} 22.1$ & VPS4B & $5^{\prime}$ \\
6B27 & Unkown & Unknown & Unknown & Unknown \\
2D18 & Unkown & Unknown & Unknown & Unknown \\
2D57 & chr22:15,889,763-15,891,681 & $22 q 11,1$ & EST & Intergenic \\
5D16 & chr5:170717291-170719525 & $5 q 35.1$ & TLX3 & 5'+body \\
5D21 & Unknown & Unknown & Unknown & Unknown \\
\hline
\end{tabular}

cisplatin-sensitive cells (T24 and KK47) and -resistant cells (T24DDP10 and KK47DDP20). In this study, RLGS was used to survey the methylation status of 1,358 genomic NotI restriction sites mainly located in CGI sequences. As a result, we detected 229 differentially methylated fragments. Seven out of 229 RLGS fragments were unmethylated in both parental cells and become methylated in cisplatin-resistant cells (Table I). Four out of 7 loci derived from our analysis were reported in a previous RLGS study (16). One loci included non-annotated gene and the residual 3 loci were 5' promoter region of SLC4A10, TLX3 and VPS4-2 ATPase. Fig. 1 shows the presence of RLGS spot 5D16 (TLX3) in T24 and KK47 cells, absence in T24DDP10 and KK47DDP20 cells.

Aberrant DNA methylation of TLX3 in cisplatin-resistant cells. Next we examined the methylation status of SLC4A10, TLX3 and VPS4-2 ATPase in 20 clinical samples of bladder cancer using the COBRA. We could not detect aberrant methylation of 5' promoter region of VPS4-2 ATPase and $S L C 4 A 10$ (data not shown). However, we detected the aberrant methylation in the 5'-promoter region of $T L X 3$ in some clinical samples of bladder cancer. Thus, we focused our analysis on the TLX3 gene. We also performed Southern blot analysis of $T L X 3$ to confirm the RLGS results in bladder cancer cells as depicted in Fig. 2A. Hybridization was performed on DNA from cisplatin-sensitive and -resistant cells digested with NotI and EcoRI, and hybridized with a portion of the RLGS sequences from spot 5D16. Lane 1 shows a normal peripheral blood leukocyte DNAs digested with EcoRI only to show the size of the EcoRI fragment. All remaining lanes show the double digest with NotI and EcoRI to see if NotI can cut the EcoRI fragment. Fig. 2B is a representative example of the Southern blot analysis. T24 and KK47 have reduced or no hybridization to the larger EcoRI fragment and stronger hybridization of the smaller NotI/EcoRI fragment, indicative of digestion and, therefore, lack of methylation of the NotI sites. In the T24DDP10 and KK47DDP20 DNAs, the hybridization signal is at the larger Not I/EcoRI fragment, indicating a lack of digestion and, therefore, hypermethylation of the NotI sites. These data serve as further evidence that the RLGS spot loss of 5D16 is indicative of hypermethylation of the NotI site found in the TLX3 gene. Furthermore, we performed the COBRA method to confirm the results above using cisplatinresistant cells and corresponding parental cells as well as several clinical samples from bladder cancer patients and

\section{$5 \mathrm{D} 16$}

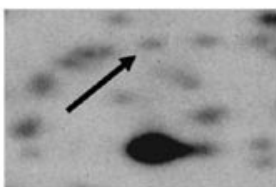

T24

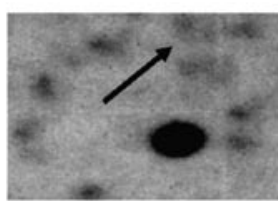

KK47
(TLX3)

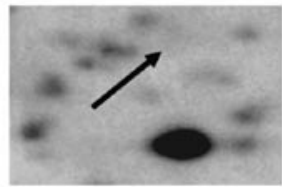

T24DDP10

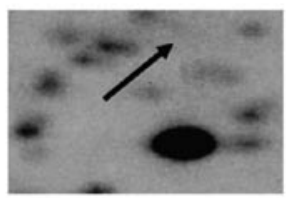

KK47DDP20
Figure 1. Representative examples of RLGS analysis for fragment 5D16 (TLX3). Sections of RLGS profiles are shown for T24, T24DDP10, KK47 and KK47DDP20. Arrowheads point to RLGS fragment 5D16 (TLX3) in parental cells (T24, KK47) and absence in cisplatin resistant cells (T24DDP10, KK47DDP20).

from their non-cancerous region. The result showed that DNAs from T24 and KK47 cells had unmethylated pattern while DNAs from their cisplatin-resistant cells had methylated pattern. In addition, DNAs from several bladder cancer samples showed methylated pattern while no DNA from non-cancerous region showed methylated pattern (Fig. 2C).

The RGLS and COBRA results of bladder cancer cells and clinical samples confirmed by bisulfite-sequencing analysis and RT-PCR. Twenty-two (21\%) out of 110 clinical samples showed the methylated pattern as evaluated by COBRA in $T L X 3$. We also performed bisulfite sequencing to detect the degree of methylation of $T L X 3$ promoter region and confirm the result of COBRA (Fig. 3A). Degree of methylation measured by bisulfite-sequencing analysis at each $\mathrm{CpG}$ site of the promoter regions was around $20 \%$ in T24 cells and tumor sample (04BT-21), these DNAs were unmethylated using COBRA in $T L X 3$. In contrast, T24DDP10 cells and tumor sample (04BT-24) which indicated the methylated pattern by COBRA showed 78.6 and $83 \%$ methylation in $T L X 3$, respectively. The methylation status of TLX3 analyzed by bisulfite sequencing was found to correlate with the results 

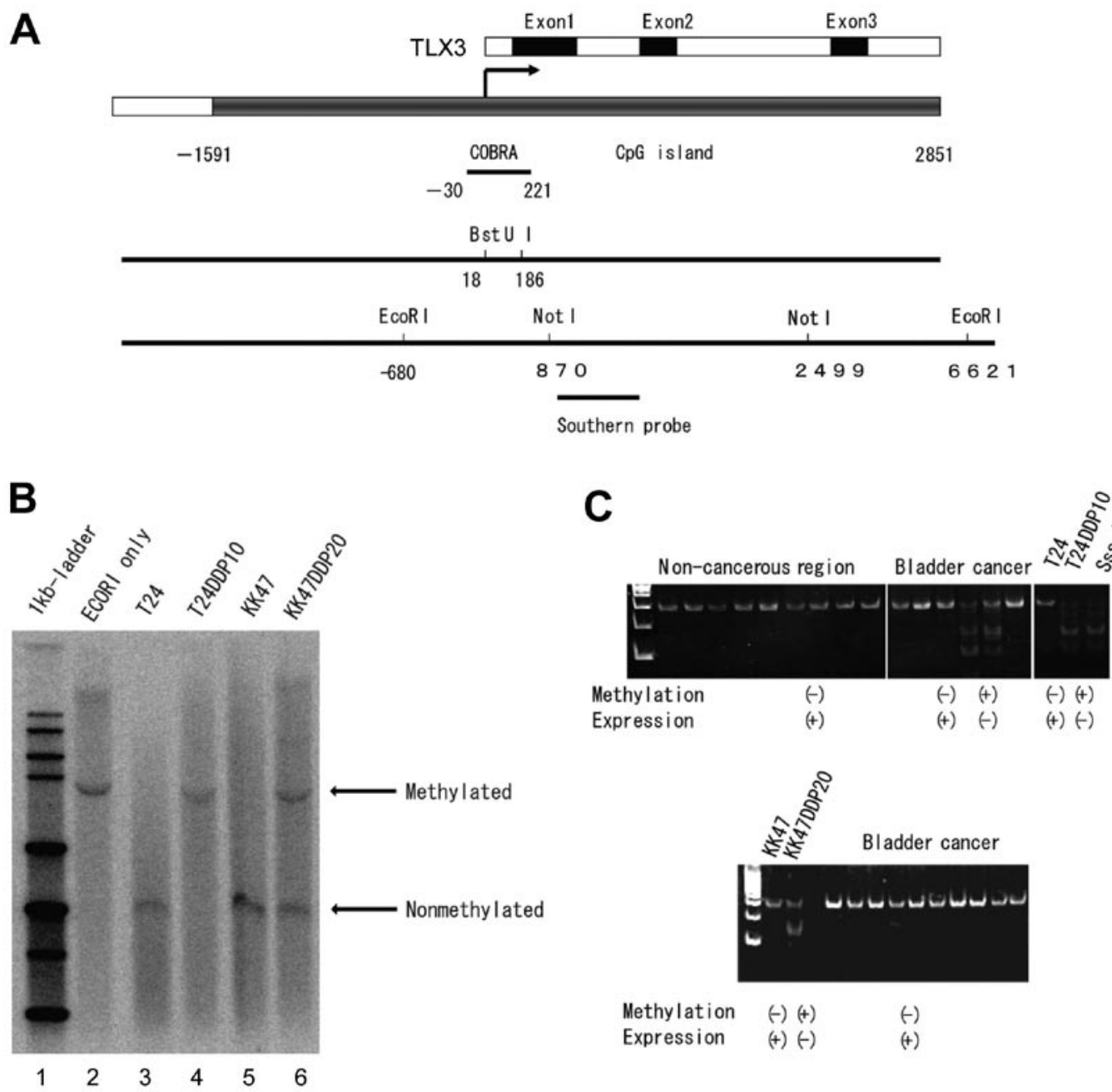

Figure 2. Methylation analysis using Southern blottng and COBRA in TLX3. (A) Schematic representation of TLX3 showing the location of the CpG island (gray bar) and the bisulfite-reaction amplicon. The arrow indicates the predicted transcription start site. The number indicates the transcriptional start site and each restriction enzyme site. (B) Southern blotting showing methylation of $T L X 3$ in bladder cancer cells. Lane 1, 1-kb ladder; lane 2, control sample (EcoRI digestion only); lane 3, T24; lane 4, T24DDP10; lane 5, KK47; lane 6, KK47DDP20 (NotI and EcoRI digested). (C) BstUI restriction digestion of bladder cancer cells results in undigested and digested fragments that indicate unmethylated and methylated DNA, respectively. SssI-treated DNA was used as methylated control and digested samples were determined as methylated DNA.

obtained by COBRA (Fig. 3A), which justifies the results of COBRA.

We also examined the TLX3 mRNA expression in cancer cells and several clinical samples from bladder cancer and non-cancerous-region using RT-PCR. As a result, we confirmed TLX3 mRNA-positive expression in T24 cells, KK47 cells and non-cancerous region, and TLX3 mRNA-negative expression in T24DDP10 cells, KK47DDP20 cells and several clinical samples from bladder cancer which showed the aberrant methylation by COBRA shown in Fig. 2C and 3B, indicating the correlation between the methylation status and gene expression of $T L X 3$.

TLX3 is involved in cisplatin resistance and cell growth. We examined whether TLX3 expression directly affects the sensitivity to cisplatin. Cisplatin-resistant cells were transiently transfected with TLX3 expression plasmid to confirm that TLX3 modulates cellular sensitivity to cisplatin. We found that overexpression of TLX3 which was confirmed by immunoblotting as shown in Fig. 4A restored cisplatin sensitivity in T24DDP10 cells using drug sensitivity assay (Fig. 4B). In contrary, cisplatin-sensitive cells originally expressing TLX3 protein were transfected with TLX3-shRNA plasmid. TLX3-knockdown which was confirmed by immunoblotting as shown in Fig. 4A conferred increased resistance against cisplatin to cisplatin-sensitive cells (Fig. 4C). These findings indicated that TLX3 expression in bladder cancer cells directly modulates cisplatin resistance.

Why did loss of TLX3 expression acquire cisplatin resistance in bladder cancer? To address this question, we also examined whether TLX3 expression modulates cell proliferation in bladder cancer using the cell proliferation assay. TLX3 overexpression in T24DDP10 cells showed increased cell proliferation compared with mock-control cells (Fig. 5A). On the other hand, TLX3-knockdown in T24 cells showed retardation of cell proliferation compared to cells transfected with control-shRNA plasmid (Fig. 5B). Thus, TLX3 expression induced the cell proliferation in bladder cancer cells, and might increase the chance that cisplatin intercalated the genomic DNA, resulting in an increase of 

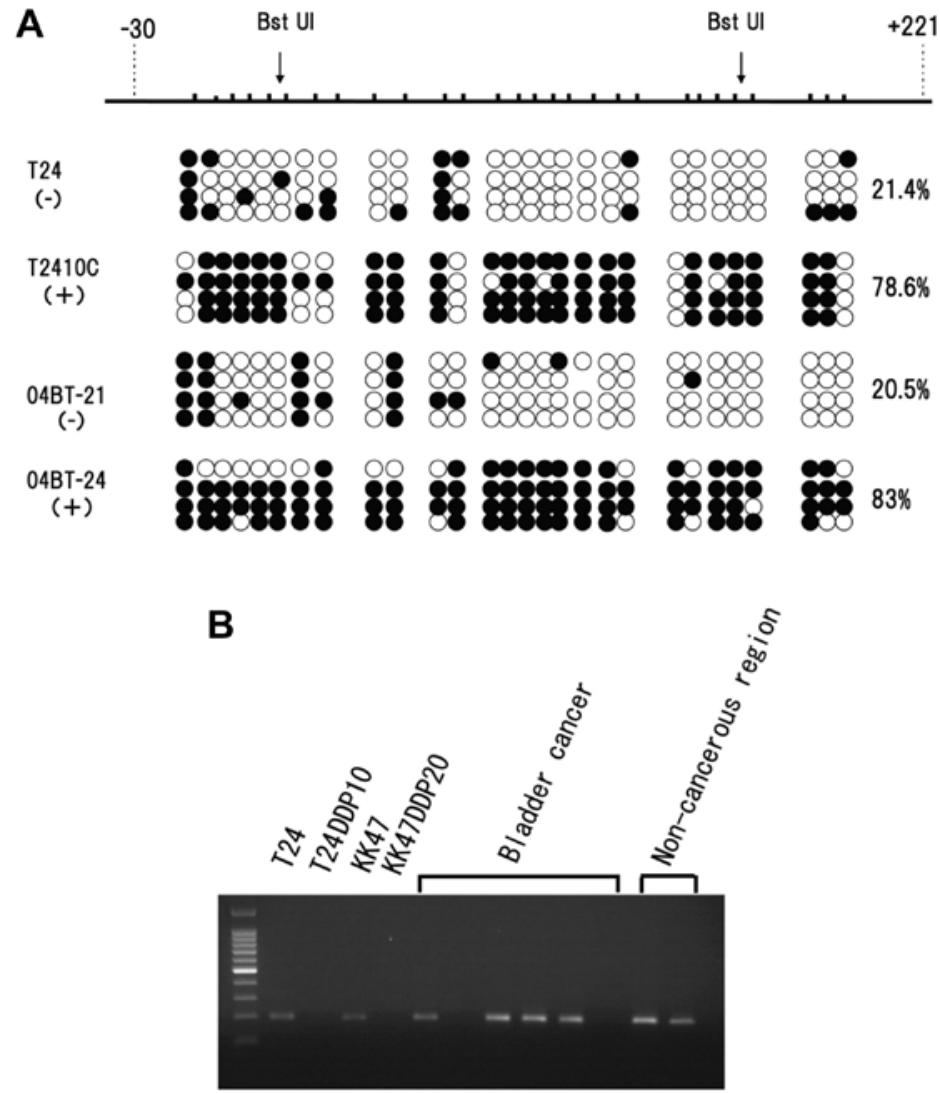

Figure 3. Methylation analysis using bisulfite sequencing and semiquantitative RT-PCR. (A) Bisulfite sequencing of $T L X 3$ in T24 cells and clinical samples from bladder cancer (04BT-21) which showed unmethylated pattern by COBRA, and in T24DDP10 cells and clinical samples from bladder cancer (04BT-24) which showed methylated pattern by COBRA. On the left, the results using COBRA; (+), hypermethylation; (-), unmethylation. Each circle represents one $\mathrm{CpG}$; closed circle, methylation; open circle, unmethylation. Arrow indicates the BstUI site and the number indicates the location from transcriptional start sites. (B) TLX3 mRNA expression by semiquantitative RT-PCR using the primers for TLX3 and GAPDH (as control, data not shown).

A

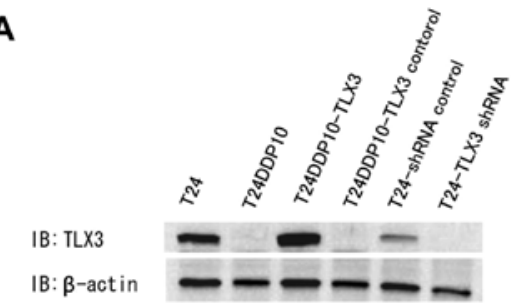

B
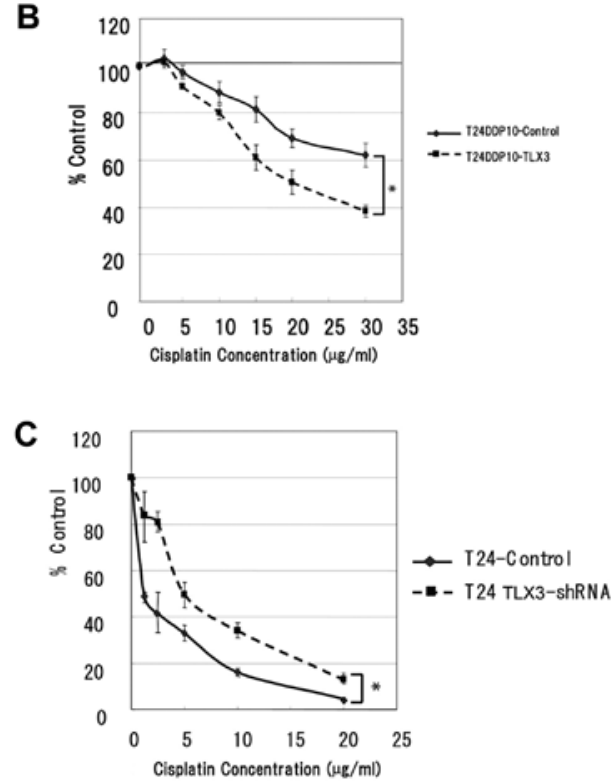

cisplatin toxicity. The loss of TLX3 expression caused the cisplatin resistance in bladder cancer cells.

Aberrant DNA methylation statuses of TLX3 in clinical samples correlate with resistance to cisplatin. We searched for correlations between $T L X 3$ methylation and drug response to cisplatin in 32 clinical samples of bladder cancer. Seventy-nine of 111 samples were too small in tumor size for the SDI test. We measured cisplatin sensitivity of 32 clinical samples by SDI test. SD activity in patients with hypermethylation of $T L X 3$ was high whereas patient samples with unmethylated $T L X 3$ showed low SD activity. This indicates a good correlation between drug resistance against cisplatin and TLX3 methylation in the clinical samples of bladder cancer (Fig. 6).

Figure 4. TLX3 expression modulates the sensitivity to cisplatin in bladder cancer cells. (A) T24 and T24DDP10 cells were transfected with the indicated shRNA $(50 \mathrm{nM})$ and expression $(0.25 \mu \mathrm{g})$ plasmids, respectively. Whole-cell extracts were subjected to SDS-PAGE, and the expression of TLX3 and $\beta$-actin was detected by Western blot analysis. (B) The transient transfection of TLX3 restores cisplatin sensitivity of T24DDP10 cells. Sensitivity to cisplatin of T24DDP10 cells transfected with CMV-control ( $\square$ ) $(0.25 \mu \mathrm{g})$, CMV-TLX3 (匹) $(0.25 \mu \mathrm{g})$ is shown. ${ }^{*} \mathrm{P}<0.05$. (C) The downregulation of TLX3-shRNA reduces cisplatin sensitivity. Sensitivity to cisplatin of T24 cells transfected with control-shRNA (ㅁ) (50 nM) and TLX3-shRNA (匹) (50 nM) is shown. ${ }^{*} \mathrm{P}<0.05$. 

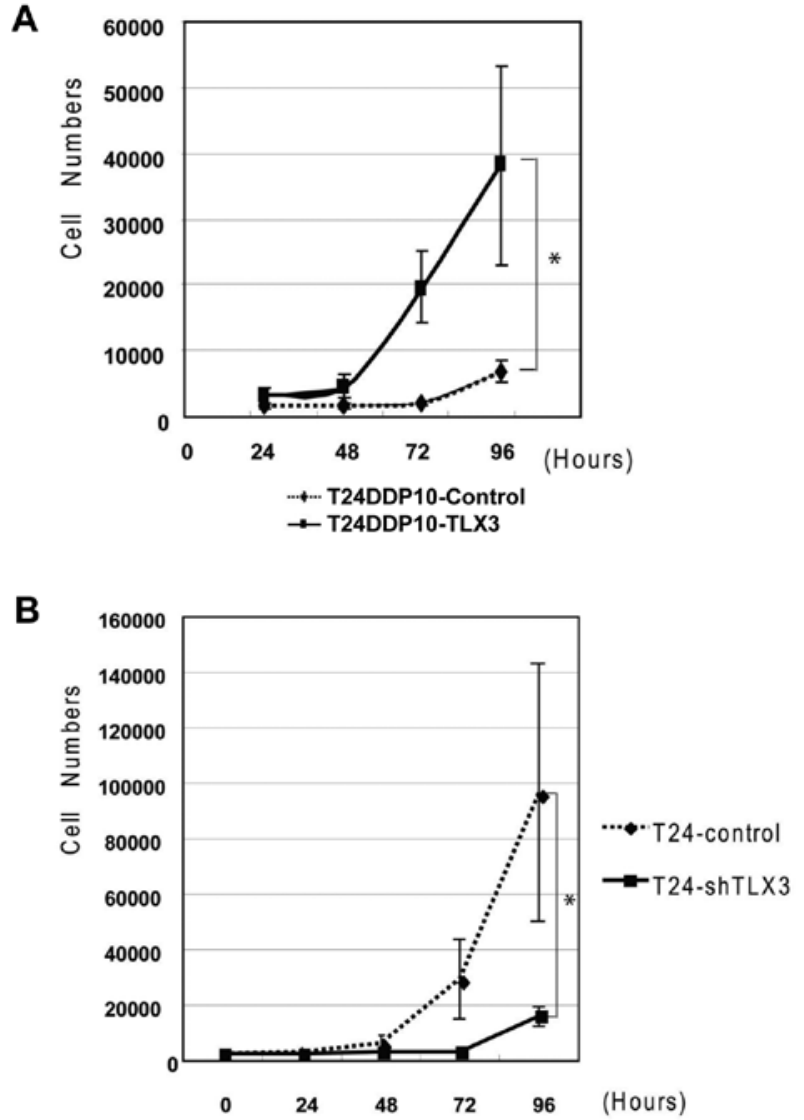

Figure 5. TLX3 expression modulates cell proliferation in bladder cancer cells. (A) Upregulation of TLX3 expression induces cell proliferation in T24DDP10. T24DDP10 cells were transiently transfected with CMV-contro () $(0.25 \mu \mathrm{g})$ or CMV-TLX3 (घ) $(0.25 \mu \mathrm{g})$. "P<0.05. (B) Knockdown of TLX3 expression suppresses cell proliferation in T24 cells. T24 cells were transiently transfected with control-shRNA () $(50 \mathrm{nM})$ or TLX3-shRNA (匹) $(50 \mathrm{nM}) .{ }^{*} \mathrm{P}<0.05$

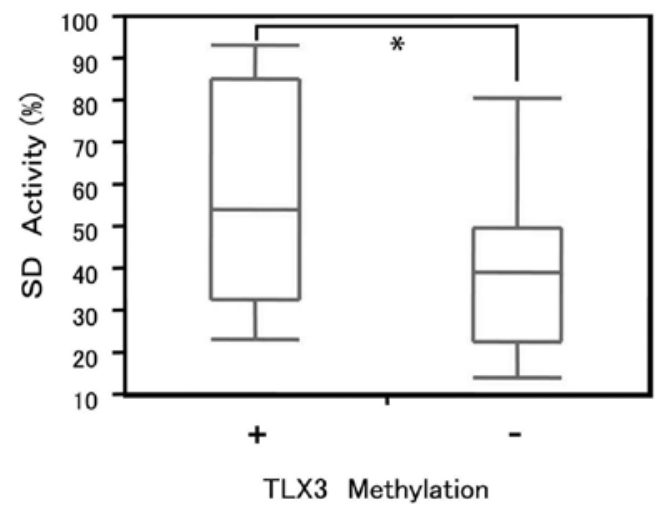

Figure 6. The correlation between methylation status of $T L X 3$ gene in clinical samples and cisplatin sensitivity. Box plot of SD activity in 32 clinical samples of bladder cancer is shown. (-), non-methylation of TLX3 $(\mathrm{n}=21) ;(+)$, hypermethylation of TLX3 $(\mathrm{n}=11)$. Middle lines, mean; upper lines, upper quartile; lower lines, lower quartile; $75 \%$; bars: \pm SD. ${ }^{*} \mathrm{P}<0.05$.

\section{Discussion}

In the present study, we analyzed potential novel biomarkers and mechanisms of cisplatin resistance in bladder cancer using bladder cancer cells and clinical samples based on DNA methylation analysis. Then, we identified $T L X 3$ as the cisplatin resistance-related gene in bladder cancer. Very little is known about $T L X 3$ gene in solid tumors, and it has not previously been associated with bladder cancer. Interestingly, however, there is evidence demonstrating that TLX3 had important roles in cell differentiation and proliferation (25-27). We showed the TLX3 was involved in cell proliferation in bladder cancer.

There is no previous report concerning the association between TLX3 and cisplatin resistance. Since the major effect of cisplatin is the formation of DNA adducts, cell-growth retardation leads to reduced opportunities for DNA to interact with cisplatin, resulting in suppression of cisplatin toxicity. TLX3 expressing cells increased sensitivity to cisplatin and negative TLX3 expressing cells decreased sensitivity to cisplatin. This statement is supported by our previous observation that cisplatin-resistant cells expressed cyclindependent kinase inhibitor p21 more abundantly and grew more slowly compared with parental cells (21). Moreover, many mechanisms of cisplatin resistance have been elucidated so far. These can be summarized as follows; increased ability of DNA repair, increased ability of the cisplatin efflux pump, increased ability of cisplatin detoxification and increased ability of anti-apoptosis (28). Although TLX3 can be classified into none of these, TLX3 might affect cisplatin resistance through the change of gene expressions because TLX 3 is a transcription factor.

We used two different bladder cancer cells, KK47 with wild-type p53 and T24 with mutated p53 (29). TLX3 methylation status in these two cells had the same pattern. Cisplatinsensitive cells showed unmethylated pattern. On the other hand, cisplatin-resistance cells showed the methylated pattern. These facts indicated that TLX3-signaling pathway for cisplatin resistance might be independent of p53 pathway. There is no evidence that cisplatin could not directly change the methylation status in $\mathrm{CpG}$ island of the genes such as 5 -azacytidine. However, conclusive molecular mechanisms of hypermethylation of $T L X 3$ gene by cisplatin have not yet been identified.

In conclusion, our findings will be useful for the discovery of new treatments through restoring sensitivity of cisplatin by modulating TLX3 expression in cisplatin-resistant cells. Cisplatin-based combination chemotherapy (GC or M-VAC) is used for bladder cancer as gold standard treatment. If we can modulate cisplatin sensitivity by TLX3 expression, patients of advanced bladder cancer will be able to receive low-dose and low-risk chemotherapy, and maintain good quality of life for longer. It would also benefit in making clinical decision to administer cisplatin-based chemotherapy or not. Aberrant TLX3 methylation in bladder cancer might be a useful diagnostic biomarker for prediction of cisplatin resistance.

\section{Acknowledgements}

This work was in part supported by Health Sciences Research Grants for Clinical Research for Evidenced Based Medicine and Grants-in-Aid for Cancer Research (016), from the Ministry of Health, Labor and Welfare, Japan; Kakenhi grants (22591769) from The Ministry of Education, Culture, Sports, Science, and 
Technology of Japan (MEXT), Japan; Research Promotion Grant from The Japanese Foundation for Prostate Research, Japan and Grant-in-Aid for Cancer Research from The Fukuoka Foundation for Sound Health, Japan. We would like to thank Ms. Noriko Hakoda, Ms. Hitomi Matoba, Ms. Miho Ushijima and Ms. Seiko Kamori for technical assistance.

\section{References}

1. Lawrence HJ and Largman C: Homeobox genes in normal hematopoiesis and leukemia. Blood 80: 2445-2453, 1992.

2. Owens BM and Hawley RG: HOX and non-HOX homeobox genes in leukemic hematopoiesis. Stem Cells 20: 364-379, 2002.

3. Wang Y, Hayakawa J, Long F, et al: 'Promoter array' studies identify cohorts of genes directly regulated by methylation, copy number change, or transcription factor binding in human cancer cells. Ann NY Acad Sci 1058: 162-185, 2005.

4. Tada Y, Wada M, Taguchi K, et al: The association of deathassociated protein kinase hypermethylation with early recurrence in superficial bladder cancers. Cancer Res 62: 4048-4053, 2002.

5. Tada Y, Wada M, Migita T, et al: Increased expression of multidrug resistance-associated proteins in bladder cancer during clinical course and drug resistance to doxorubicin. Int J Cancer 98: 630-635, 2002.

6. Tada Y, Brena RM, Hackanson B, Morrison C, Otterson GA and Plass C: Epigenetic modulation of tumor suppressor CCAAT/ enhancer binding protein alpha activity in lung cancer. J Natl Cancer Inst 98: 396-406, 2006.

7. Shen Y, Chow J, Wang Z and Fan G: Abnormal CpG island methylation occurs during in vitro differentiation of human embryonic stem cells. Hum Mol Genet 15: 2623-2635, 2006.

8. Vucic EA, Brown CJ and Lam WL: Epigenetics of cancer progression. Pharmacogenomics 9: 215-234, 2008.

9. Li LC, Carroll PR and Dahiya R: Epigenetic changes in prostate cancer: implication for diagnosis and treatment. J Natl Cancer Inst 97: 103-115, 2005.

10. Kalebic T: Epigenetic transitions: towards therapeutic targets. Expert Opin Ther Targets 7: 693-699, 2003.

11. Kim WJ and Kim YJ: Epigenetic biomarkers in urothelial bladder cancer. Expert Rev Mol Diagn 9: 259-269, 2009.

12. Nishiyama N, Arai E, Chihara Y, et al: Genome-wide DNA methylation profiles in urothelial carcinomas and urothelia at the precancerous stage. Cancer Sci 101: 231-240, 2010.

13. Wang SS, Smiraglia DJ, Wu YZ, et al: Identification of novel methylation markers in cervical cancer using restriction landmark genomic scanning. Cancer Res 68: 2489-2497, 2008.

14. Smiraglia DJ, Smith LT, Lang JC, et al: Differential targets of $\mathrm{CpG}$ island hypermethylation in primary and metastatic head and neck squamous cell carcinoma (HNSCC). J Med Genet 40: 25-33, 2003.

15. Park J, Brena RM, Gruidl M, et al: $\mathrm{CpG}$ island hypermethylation profiling of lung cancer using restriction landmark genomic scanning (RLGS) analysis. Cancer Biomark 1: 193-200, 2005.
16. Kotoh S, Naito S, Yokomizo A, Kohno K, Kuwano M and Kumazawa J: Enhanced expression of gamma-glutamylcysteine synthetase and glutathione S-transferase genes in cisplatin-resistant bladder cancer cells with multidrug resistance phenotype. J Urol 157: 1054-1058, 1997.

17. Kotoh S, Naito S, Yokomizo A, et al: Increased expression of DNA topoisomerase I gene and collateral sensitivity to camptothecin in human cisplatin-resistant bladder cancer cells. Cancer Res 54: 3248-3252, 1994.

18. Dai Z, Lakshmanan RR, Zhu WG, et al: Global methylation profiling of lung cancer identifies novel methylated genes. Neoplasia 3: 314-323, 2001.

19. Smiraglia DJ, Fruhwald MC, Costello JF, et al: A new tool for the rapid cloning of amplified and hypermethylated human DNA sequences from restriction landmark genome scanning gels. Genomics 58: 254-262, 1999.

20. Herman JG, Graff JR, Myohanen S, Nelkin BD and Baylin SB: Methylation-specific PCR: a novel PCR assay for methylation status of CpG islands. Proc Natl Acad Sci USA 93: 9821-9826, 1996.

21. Nagel S, Kaufmann M, Drexler HG and MacLeod RA: The cardiac homeobox gene NKX2-5 is deregulated by juxtaposition with BCL11B in pediatric T-ALL cell lines via a novel $t(5 ; 14)$ (q35.1;q32.2). Cancer Res 63: 5329-5334, 2003.

22. Shiota M, Yokomizo A, Kashiwagi E, et al: Foxo3a expression and acetylation regulate cancer cell growth and sensitivity to cisplatin. Cancer Sci 101: 1177-1185, 2010.

23. Shiota M, Yokomizo A, Tada Y, et al: P300/CBP-associated factor regulates Y-box binding protein-1 and promotes cancer cell growth, cancer invasion and drug resistance. Cancer Sci 101: 1797-1806, 2010.

24. Maehara Y, Anai H, Kusumoto H and Sugimachi K: Poorly differentiated human gastric carcinoma is more sensitive to antitumor drugs than is well differentiated carcinoma. Eur J Surg Oncol 13: 203-206, 1987.

25. Su X, Drabkin H, Clappier E, et al: Transforming potential of the T-cell acute lymphoblastic leukemia-associated homeobox genes HOXA13, TLX1, and TLX3. Genes Chromosomes Cancer 45: 846-855, 2006.

26. Shi Y, Chichung Lie D, Taupin P, et al: Expression and function of orphan nuclear receptor TLX in adult neural stem cells. Nature 427: 78-83, 2004.

27. Nagel S, Venturini L, Przybylski GK, et al: Activation of miR-17-92 by NK-like homeodomain proteins suppresses apoptosis via reduction of E2F1 in T-cell acute lymphoblastic leukemia. Leuk Lymphoma 50: 101-108, 2009.

28. Kohno K, Uchiumi T, Niina I, et al: Transcription factors and drug resistance. Eur J Cancer 41: 2577-2586, 2005.

29. Hinata N, Shirakawa T, Zhang Z, et al: Radiation induces p53-dependent cell apoptosis in bladder cancer cells with wildtype-p53 but not in p53-mutated bladder cancer cells. Urol Res 31: 387-396, 2003. 